\title{
Aqueous Two-Phase System Patterning of Microbubbles: Localized Induction of Apoptosis in Sonoporated Cells
}

\author{
John P. Frampton, Zhenzhen Fan, Arlyne Simon, Di Chen, Cheri X. Deng,** \\ and Shuichi Takayama*
}

Ultrasound-driven microbubbles produce mechanical forces that can disrupt cell membranes (sonoporation). However, it is difficult to control microbubble location with respect to cells. This lack of control leads to low sonoporation efficiencies and variable outcomes. In this study, aqueous two-phase system (ATPS) droplets are used to localize microbubbles in select micro-regions at the surface of living cells. This is achieved by stably partitioning microbubbles in dextran (DEX) droplets, deposited on living adherent cells in medium containing polyethylene glycol (PEG). The interfacial energy at the PEG-DEX interface overcomes microbubble buoyancy and prevents microbubbles from floating away from the cells. Spreading of the small DEX droplets retains microbubbles at the cell surface in defined lateral positions without the need for antibody or cell-binding ligand conjugation. The patterned microbubbles are activated on a cell monolayer exposed to a broadly applied ultrasound field (center frequency $1.25 \mathrm{MHz}$, active element diameter $0.6 \mathrm{~cm}$, pulse duration $8 \mu \mathrm{s}$ or $30 \mathrm{~s}$ ). This system enables efficient testing of different ultrasound conditions for their effects on sonoporation-mediated membrane disruption and cell viability. Regions of cells without patterned microbubbles show no injury or membrane disruption. In microbubble patterned regions, $8 \mu$ s ultrasound pulses $(0.2-0.6 \mathrm{MPa})$ produce cell death that is primarily apoptotic. Ultrasound-induced apoptosis increases with higher extracellular calcium concentrations, with cells displaying all of the hallmarks of apoptosis including annexinV labeling, loss of mitochondrial membrane potential, caspase activation and changes in nuclear morphology.

stenciling and inkjet printing ${ }^{[1]}$ ) or by localizing reagents over a preexisting cell monolayer (e.g., laminar flow patterning ${ }^{[2,3]}$ or microelectromechanical system (MEMS)based culture platforms ${ }^{[4,5]}$. However, these strategies can be difficult to translate to wide-spread use in the life sciences because they often use custom fabricated devices that require a high degree of user expertise to operate.

Recently, we developed a microdevicefree technology that can pattern cells and deliver biomolecular treatments to specific populations of cells. This user-friendly, cell-compatible approach involves confinement of biomolecules within the dextran (DEX) phase of a polyethylene glycol (PEG)/DEX aqueous twophase system (ATPS) ${ }^{[6,7]}$ ATPS printing is non-destructive to cells, easy to perform, inexpensive and can be used with many types of cell culture systems. In addition, ATPSs can be used to directly pattern particles of various sizes including viruses, bacteria and mammalian cells, all of which are not easily patterned with other methods. ${ }^{[7-10]}$

We predicted that lipid coated, gas core microbubbles could also be patterned using ATPS. When driven by ultrasound,

\section{Introduction}

Cell and biomolecule patterning is used in tissue engineering and biomedical research for high-throughput cell assays and investigation of interactions among populations of cells. Patterning is typically achieved by depositing materials on a substrate prior to cell attachment (e.g., microcontact printing,

Dr. J. P. Frampton, Dr. Z. Fan, Dr. D. Chen,

Prof. C. X. Deng, * Prof. S. Takayama

Department of Biomedical Engineering

University of Michigan

Ann Arbor, USA

E-mail: cxdeng@umich.edu; takayama@umich.edu

A. Simon

Department of Macromolecular Science and Engineering

University of Michigan

Ann Arbor, USA

DOI: 10.1002/adfm.201203321 microbubbles rapidly expand and contract, and/or collapse, producing submicron or micron scale mechanical effects including high speed fluidic microjets, shear stress, shock waves and direct deformation of cell membranes. ${ }^{[11-15]}$ These mechanical forces can create pores in cell membranes, that can be transient or irreversible depending on the ultrasound parameters. The membrane disruption mediated by ultrasound excitation of microbubbles, referred to as sonoporation, has found applications both in vitro and in vivo, emerging as a valuable tool for transiently disrupting cell membranes to allow entry of membrane impermeable exogenous agents into cells or to induce cell death. ${ }^{[14,16,17]}$ This technique is particularly advantageous because ultrasound application is noninvasive. However, sonoporation is limited by a lack of control over microbubble location with respect to cells, often resulting in variable treatment outcomes and suboptimal efficiencies. Gas-filled microbubbles easily float to the surface of the cell culture medium in vitro, far from where they can exert their effects on adherent cells. Even 
in cases where microbubbles are mixed in solution with suspended cells, the temporal variation of their spatial distribution in the bulk solution caused by buoyancy results in unpredictable outcomes, precluding detailed investigation of pore formation and cell injury/death.

In ultrasound molecular imaging, much effort has been devoted to developing strategies for decorating the exterior of encapsulated microbubbles with antibodies/ligands that target them to the surface of cells. ${ }^{[18-21]}$ Since these targeted microbubbles attach to cell membranes, they can also provide the opportunity to disrupt cell membranes. ${ }^{[22-24]}$ For in vitro experiments, binding of targeted microbubbles to adherent cells is typically achieved by applying a small amount of solution containing the targeted microbubbles to the cells in a cell culture dish or to cells growing within a specialized chamber. In these examples, the microbubbles bind to the entire cell monolayer; therefore, sonoporation is induced in all cells with bound microbubbles. Such systems do not provide the spatial selection that is required to target subpopulations of cells within a monolayer. Furthermore, expensive antibodies/ligands are needed to attach the microbubbles to the cells.

In this study, we developed a novel approach to address these limitations by spatially patterning microbubbles using ATPSs and confining them to the surface of cells, where they can be subsequently excited by ultrasound. This approach allows localization of ultrasound-driven microbubbles to specific sites within a cell culture system, which cannot be achieved by any other currently available method. Furthermore, our system is compatible with most types of cell culture substrates including standard Petri dishes and well plates made from tissue culture plastics. The patterned microbubbles allow both the experimental treatments and the control (where cells within different regions are exposed to the same ultrasound condition without microbubbles nearby) to be simultaneously performed, in one step, in a single cell culture dish. By patterning microbubbles at multiple sites, multiple ultrasound conditions can be tested within the same culture dish, providing a multiplexed platform for efficient investigation of ultrasound-mediated sonoporation and downstream effects on cells, including induction of apoptosis. Our approach provides an easy method for targeting cells because it does not require expensive antibodies or ligands for decorating the microbubbles.

\section{Results}

\subsection{Microbubble Patterning}

We achieved microbubble patterning by dispensing droplets of DEX containing microbubbles through a glass capillary needle onto a cell monolayer immersed in PEG. Prior to dispensing the microbubbles, we broke the end of the capillary to prevent clogging and shearing of bubbles during the dispensing process. The use of a capillary needle allowed us to dispense droplets of DEX $(\sim 0.05-0.10 \mu \mathrm{L})$ that were much smaller than droplets produced using a micropipettor $(0.5-2.0 \mu \mathrm{L})$ (Figure 1A-C). DEX has a slightly greater density than PEG, causing the DEX droplets to sink and contact the cell monolayer. Spreading of the small DEX droplets over the cells confined the microbubbles at the cell surface (Z-dimension). This method allowed us to place multiple, discrete or continuous treatment sites within the same monolayer (XY dimensions) without the use of specialized fluidic devices, membrane plates or dish inversion. After patterning the DEX droplets, additional PEG was added to the dish without disturbing the patterning so that the active element at the tip of the ultrasound transducer (which was focused on the cells on the dish) was immersed. Application of ultrasound was then used to excite the microbubbles and generate cavitation, or rapid expansion and contraction of the microbubbles, resulting in bursting or shrinking of the microbubbles near the cells (Figure 1D-F), confirming that the microbubbles in DEX behaved similarly as they do in cell culture medium. ${ }^{[25]}$ It was possible to dispense droplets of DEX containing microbubbles in close proximity to one another for multiplexing and to generate a variety of specialized user-defined patterns (Figure 1G). Ultrasound excitation of the patterned microbubbles generated membrane disruption and induced cell death in discrete regions, as indicated by the staining pattern for annexinV (apoptosis) and propidium iodide (PI) (membrane disruption) in the microbubble patterned regions (Figure $1 \mathrm{H}$ ).

We found that patterning was dependent on the type of microbubbles due to differential partitioning in the ATPS. We tested two types of commercially available microbubbles, Definitylipid coated microbubbles and Targestar-SA microbubbles (with and without antibody conjugation), to determine their partitioning in the PEG/DEX system (Figure 2A). Definity microbubbles partitioned primarily to the PEG phase and to the interface for the phase systems we tested. This occurred because the non-polar lipid shells of the Definity microbubbles were more compatible with PEG. Targestar-SA microbubbles partitioned primarily to the interface and to the DEX phase. A greater proportion of microbubbles were observed in the DEX phase for lower DEX molecular weights (3500 and $10000 \mathrm{kDa}$ DEX), most likely because proteins such as streptavidin display stronger partitioning to DEX at low DEX molecular weights. This was true regardless of the presence of conjugated antibody. Partitioning to the interface was not only dependent on microbubble chemistry and size, but was also caused by buoyancy of the microbubbles in DEX.

Microbubble partitioning was confirmed by placing microbubbles directly into DEX and then dispensing DEX/microbubble droplets into a solution of PEG (Figure 2B). Definity microbubbles rapidly moved to the interface, with some crossing the interface and entering the PEG. In contrast, Targestar-SA microbubbles remained well distributed in the DEX droplet. These experiments allowed us to monitor the distribution of microbubbles in DEX droplets and showed that Targestar-SA microbubbles remained in the DEX droplets in spite of their buoyancy.

We next tested the ability of various DEX/microbubble combinations to disrupt cell membranes upon ultrasound application in the presence of the cell impermeable dye PI (Figure 2C). As expected, no PI staining was observed with ultrasound application to patterned DEX droplets using Definity microbubbles, as these microbubbles rapidly escaped from the DEX phase and floated to the surface of the PEG, where they had no effect on the cells. Patterned droplets containing Targestar-SA 


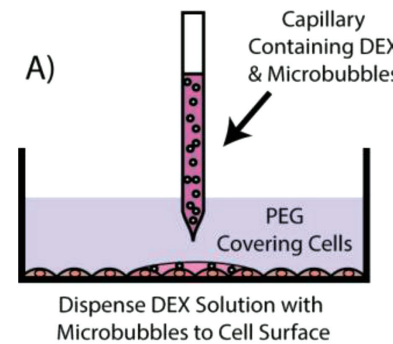

Microbubbles to Cell Surface

B)

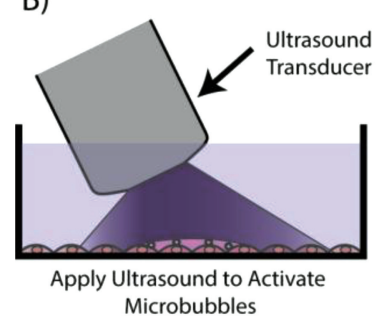

C)

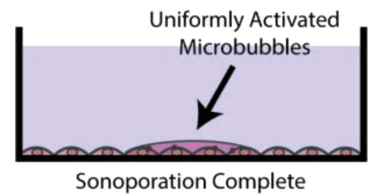

D) Patterned Microbubbles Before Ultrasound

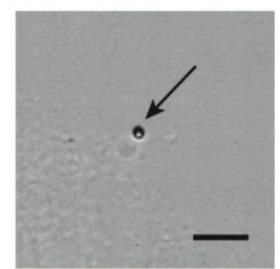

E) During Ultrasound

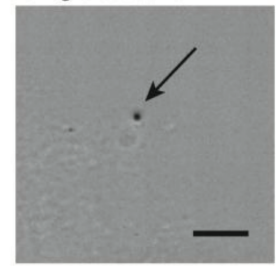

F) After Ultrasound

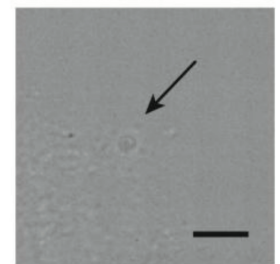

G)

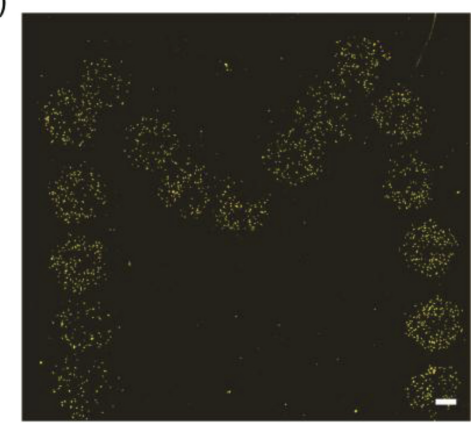

H)

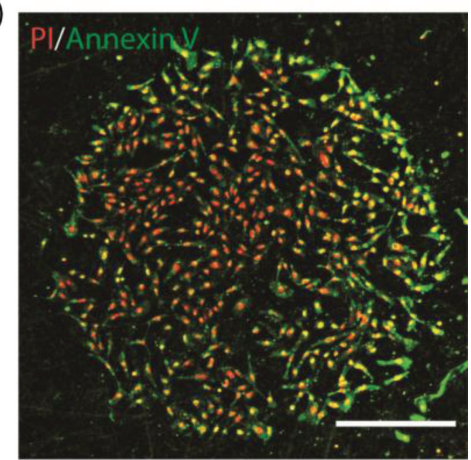

Figure 1. Patterned sonoporation is achieved by depositing microbubbles on cells in the DEX phase of a PEG/DEX ATPS. A) Microbubbles are dispensed onto cells in PEG using a glass capillary needle. B,C) The ultrasound transducer is used to activate the bubbles which are contained within the DEX phase. D-F) High magnification images of a single microbubble in DEX attached to a single cell (as indicated by the black arrows) before, during, and after ultrasound application captured with a high speed camera. This bubble was activated upon ultrasound treatment, undergoing rapid expansion and contraction, which resulted in bursting/shrinking on the cell, as visualized using ultrafast videomicroscopy. As microbubbles expand and contract during ultrasound application at $1.25 \mathrm{MHz}$, halos appear around the microbubbles due to rapid volume changes (E). After microbubble activation, the microbubbles disappear or become smaller in size (F). G) Microbubbles can be patterned with various user-defined array geometries, as indicated by patterning of a Michigan "M", visualized with intracellular PI staining after microbubble excitation. H) Apoptosis was selectively induced in the microbubble patterned regions treated with ultrasound ( $0.6 \mathrm{MPa}, 8 \mu$ s pulse) as indicated by annexinV (green) and PI (red) staining after ultrasound treatment. All images are for non-conjugated Targestar-SA microbubbles. Scale bars are $\sim 5 \mu \mathrm{m}$ in $(\mathrm{D}-\mathrm{F})$ and $\sim 300 \mu \mathrm{m}$ in $(\mathrm{G})$ and $(\mathrm{H})$.

microbubbles, however, were able to disrupt cell membranes allowing entry of PI into cells in the DEX/microbubble-covered regions, even without antibody conjugation of the microbubbles to the cells. We did not notice a difference in the number of PI loaded cells following ultrasound treatment as a function of antibody concentration (i.e., with or without anti-CD31 antibody which targets HUVECs), indicating that the unattached microbubbles had an effect on cells by virtue of their close proximity to the cell surface, as dictated by the small height of the DEX droplet. This finding is important because it demonstrates the ability of micropatterned DEX droplets to spatially confine the microbubbles to the cell surface for sonoporation without the need for coupling to expensive antibodies.

For subsequent experiments we chose to use the DEX $10000 \mathrm{kDa} / \mathrm{PEG} 35000 \mathrm{kDa}$ phase system with unconjugated Targestar-SA microbubbles, since this formulation displayed reasonable partitioning properties, and had polymer concentrations and viscosities low enough for use with cells. In addition, DEX $10000 \mathrm{kDa}$ showed more spreading on cells than DEX $500000 \mathrm{kDa}$, ensuring that bubbles floating to the interface would still remain in close proximity to the cells.

\subsection{Sonoporation of Sub-Populations of Cells within a Monolayer: Effects of Polymer Concentration, Microbubble Concentration and Cell Type}

We applied our DEX/microbubble patterning system to investigate cell membrane disruption and death as a function of ultrasound pressure, polymer concentration, microbubble concentration and cell type. We used PI (applied after ultrasound) as a marker for cell membrane disruption and cell death. Since microbubbles could be arrayed with various spacing and number of treated regions, this allowed us to test multiple ultrasound conditions in parallel (e.g., different numbers of pulses and pressure amplitudes), essentially multiplexing the ultrasound treatment (Figure 3A). Without ATPS localization (spatial scale of $\sim 0.75 \mathrm{~mm}^{2}$ or less with ATPS), this type of multiplexing would not have been possible, since the ultrasound excitation covers a treatment area several times larger than the ATPS microbubble patterns.

We next tested different polymer concentrations of DEX in the 16\% PEG $35000 \mathrm{kDa} / \mathrm{DEX} 10000 \mathrm{kDa}$ system. Both the dextran and the PEG used in our system are biocompatible 
A)

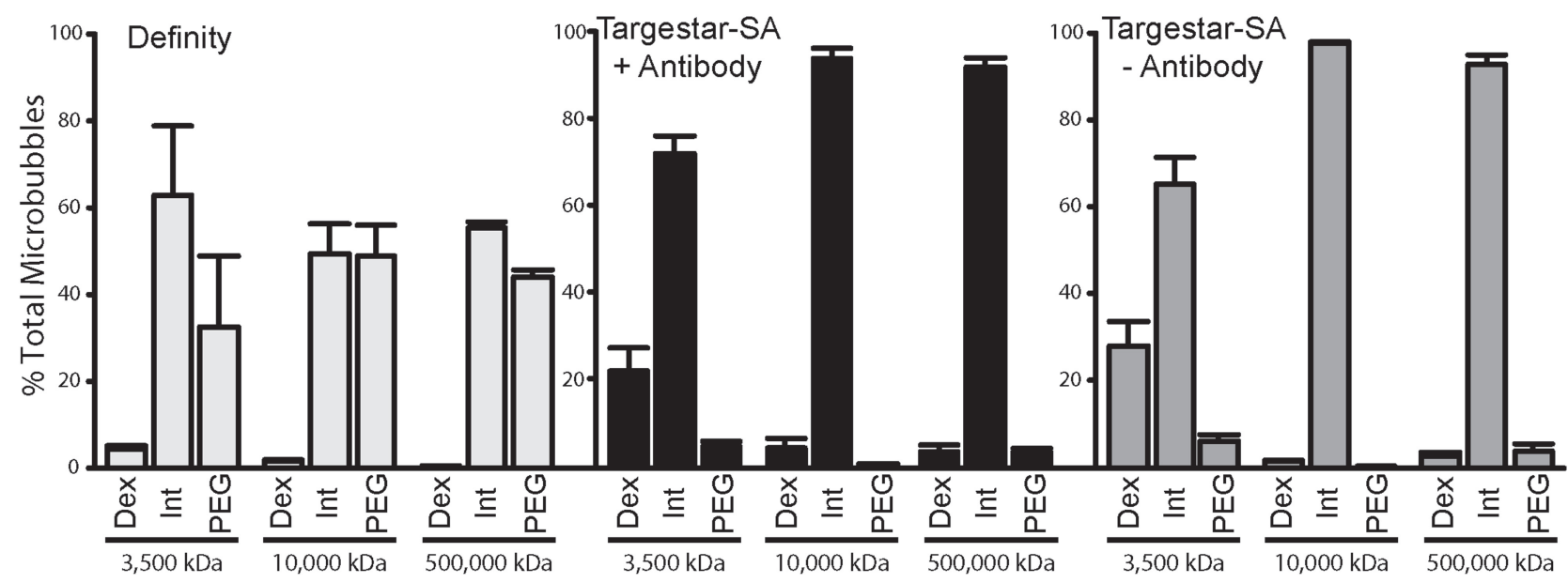

B)

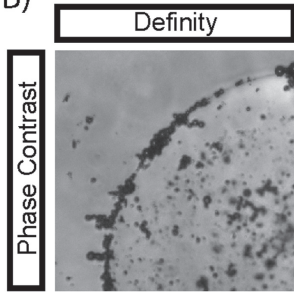

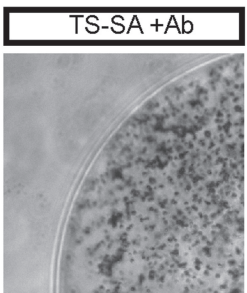

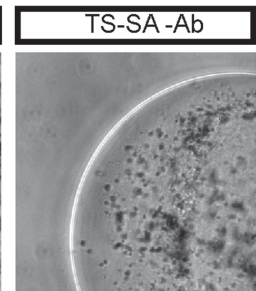

C)

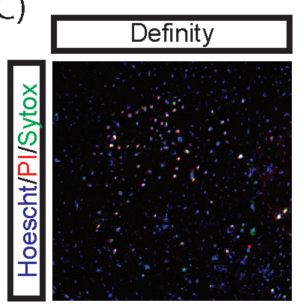

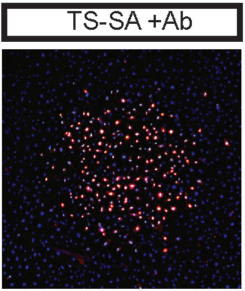

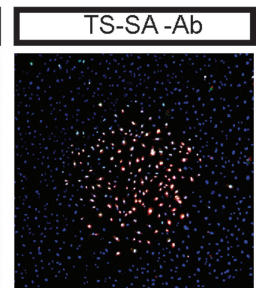

Figure 2. Partitioning of microbubbles. A) The percentage of microbubbles present in DEX, at the PEG/DEX interface and in PEG was calculated for various ATPS formulations with different DEX molecular weights. Definity microbubbles localized to the interface and the PEG phase, whereas Targestar-SA microbubbles localized to the interface and the DEX phase for lower molecular weight of DEX. Bars denote mean \pm SEM. B) Microbubbles that were deposited within DEX droplets and tracked by video microscopy confirmed the results in (A). Definity bubble collected at the PEG/DEX interface and eventually crossed the interface to enter the PEG phase. Targestar-SA microbubbles were well-retained in DEX. Scale bar $\sim 60 \mu \mathrm{m}$. C) Droplets of DEX containing Definity microbubbles could not mediate transmembrane delivery of PI in HUVECs. Droplets with Targestar-SA microbubbles mediated PI delivery with high efficiency in HUVECs, even without antibody targeting. Scale bar $\sim 300 \mu \mathrm{m}$.

and noncytotoxic; therefore, the polymers themselves had little to no direct effect on cell apoptosis. Moreover, the intracellular cytocompatibility of these polymers has been demonstrated using lipofection as a membrane disruption method, as well as through numerous studies that have used cell microinjection of fluorophore-conjugated polymers such as dextran for cell tracing. For these experiments, a single ultrasound pulse was applied $(8 \mu$ s duration and $0.4 \mathrm{MPa}$ peak negative pressure) to excite the microbubbles. A DEX concentration of $16 \%$ was optimal for maintaining an even distribution of microbubbles on the cells. Solutions of $8 \%$ DEX and 4\% DEX produced clusters of microbubbles on the cells, resulting in uneven microbubble distribution, inefficient microbubble activation and reduced efficiency of cell membrane disruption (Figure 3B). The clustering effect was likely due to equilibration of the DEX droplets in PEG, which caused the DEX solution to decrease dramatically in volume, resulting in microbubble aggregation.

Using the 16\% PEG $35000 \mathrm{kDa} / 16 \%$ DEX $10000 \mathrm{kDa}$ system, we tested the effect of microbubble concentration on membrane disruption. The highest concentration of microbubbles we tested was $\sim 0.5 \times 10^{9} / \mathrm{mL}$, which was determined by the maximum concentration of microbubbles provided by the manufacturer $\left(\sim 1.0 \times 10^{9} / \mathrm{mL}\right)$ and the maximum concentration of DEX $10000 \mathrm{kDa}$ that could be easily prepared $(32 \%)$ mixed in equal parts $(0.5 \mathrm{X}$ microbubble condition). We also tested microbubble concentrations that were 10 -fold and 100 -fold lower $(0.05 \mathrm{X}$ and $0.005 \mathrm{X}$ microbubble conditions). The efficiency of cell membrane disruption decreased dramatically as microbubble concentration decreased (Figure 3C).

It should be noted that the microbubble concentration we used $\left(\sim 0.5 \times 10^{9} / \mathrm{mL}\right)$ was much higher than what is typically used for sonoporation. The small volumes of our ATPS droplets made it possible for us to use such high concentration of microbubbles in our study. This would be prohibitive in a conventional sonoporation experiment due to the cost associated with the large amount of this reagent. Although it is expected that high bubble concentrations should generate the strongest sonoporation effects on cells, we have observed interactions among microbubbles (due to the secondary radiation force or Bjerknes force) at high microbubble concentrations. ${ }^{[26,27]}$ This increased level of microbubble-microbubble interaction may influence their ability to produce direct effects on the cells during sonoporation. Thus, attempts to use microbubble concentrations much higher than those 
A)

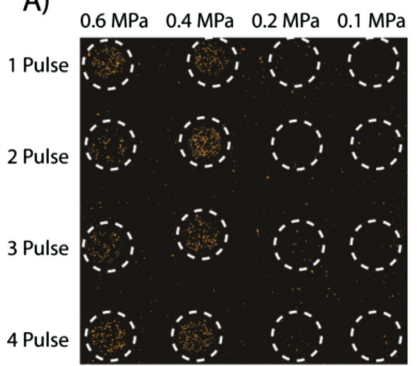

E)

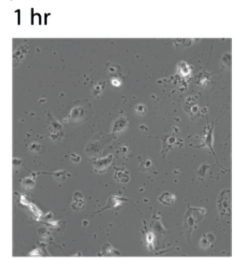

$24 \mathrm{hr}$

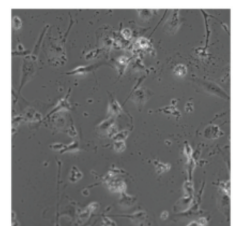

B)

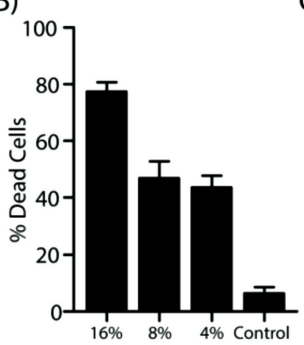

DEX Concentration (\% wt.)

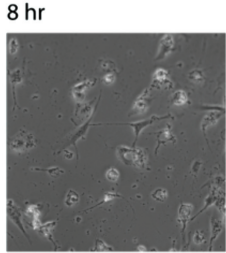

$28 \mathrm{hr}$

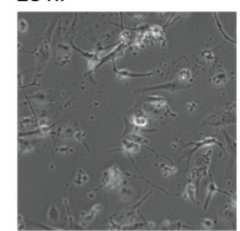

$4 \mathrm{hr}$

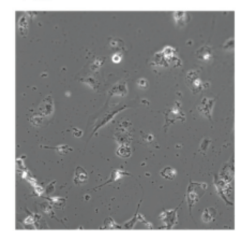

$32 \mathrm{hr}$

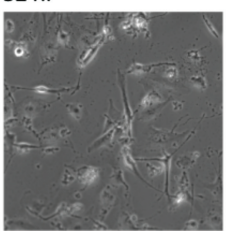

C)

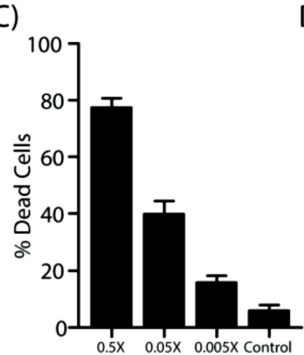

Microbubble Concentration

$12 \mathrm{hr}$

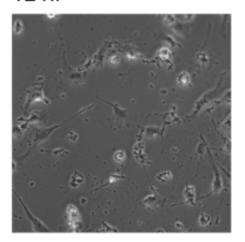

$36 \mathrm{hr}$

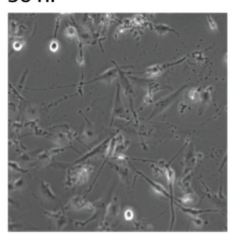

D)

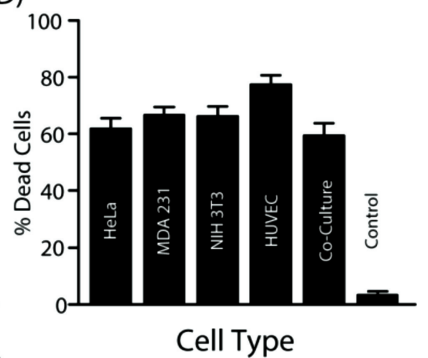

$16 \mathrm{hr}$
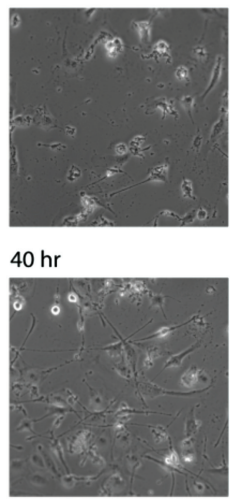

$20 \mathrm{hr}$

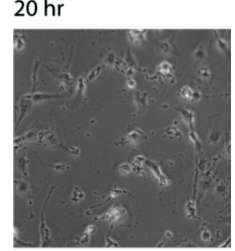

$44 \mathrm{hr}$

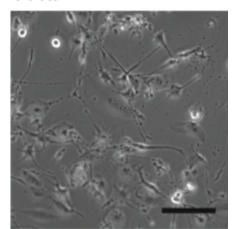

Figure 3. Optimization of ultrasound and microbubble conditions for targeted cell killing. A) Placing DEX droplets in an array allows multiplexed testing of ultrasound parameters such as number of pulses (down) and pressure amplitude (across). The array image is a reconstructed mosaic from high magnification brightfield and PI images. Scale $\sim 500 \mu \mathrm{m}$. Cell death was minimal at low pressures (0.1 and $0.2 \mathrm{MPa})$. Multiple low pressure pulses (3 or 4 pulses) resulted in a slight increase in cell death for the $0.2 \mathrm{MPa}$ ultrasound condition. Pressures of 0.4 and $0.6 \mathrm{MPa}$ resulted in a high proportion of cells dying in the patterned areas, with little effect from additional pulses. B) Decreasing the DEX concentration resulted in microbubble aggregation. Cell death was markedly reduced at $8 \%$ and $4 \%$ DEX concentrations. C) The proportion of dead cells decreased with decreasing microbubble concentrations. D) HUVECs were most sensitive to microbubble excitation, but cell death was similar among all the cell types we tested. Control regions without microbubbles showed minimal cell death (data compiled for all cell types). Bars in (B-D) denote mean \pm SEM for $n=3$ independent experiments. E) Time lapse imaging of microbubble targeted co-cultures of MDA231, NIH 3T3 and HUVECs showed that cell debris was gradually removed over the course of several days as the treated regions became repopulated by cells from surrounding untreated areas. Scale $\sim 60 \mu \mathrm{m}$.

used in this study are unlikely to further enhance the efficiency of sonoporation.

Finally, we examined ultrasound-induced membrane disruption in different cell types using the $0.5 \mathrm{X}$ microbubble condition and the 16\% PEG 35,000 kDa/16\% DEX 10,000 kDa system. Of the five cell types we tested, (HUVEC, NIH3T3, MDA 231, HeLa and HUVEC/NIH3T3/MDA231 co-cultures), HUVECs were most sensitive to membrane disruption, while the overall level of cell death was similar among all other cell types (Figure 3D). These results were expected because HUVECs, as with other types of primary cells, are typically more sensitive to physical or chemical disruption than immortalized cell lines and cancer cells. Cell death was less than $5 \%$ in control regions without microbubbles for all of the cell types we tested. Time lapse imaging of the ultrasound treated microbubble sites revealed that dead cells remained in place after treatment. Over the course of $48 \mathrm{~h}$, surviving cells from the treated regions and healthy cells from the surrounding regions frequently interrogated the dead cells (Figure 3E, Supporting Information Video 1), eventually displacing the dead cells through active removal of cell debris, migration and proliferation.

\subsection{Platforms for Testing Cell Physiology: Apoptosis}

Most platforms for wound healing research involve either physical damage/displacement or chemical ablation that completely removes the cells. ${ }^{[28-31]}$ In these assays, it is possible to quantify cell migration into cleared regions and secondary damage in the surrounding regions. However, many types of injuries in vivo do not result in complete removal of cells from the tissue all at once. Rather, cells undergo a more complex removal process where they die from direct trauma (necrosis) or initiate their own death in response to unfavorable environmental factors (apoptosis) and must then be cleared by immune cells in the surrounding tissue. Thus, a physiological model for tissue damage should involve both necrotic and apoptotic modes of cell death, where the damaged/dying cells remain in place throughout the process. As demonstrated by the results presented in Figure 3E and Supporting Information Video 1, ultrasound excitation of patterned microbubbles can effectively generate cells death in targeted regions without removal of cells. Patterning microbubbles to control the location of this injury provides an ideal platform for investigating the physiology of the damaged cells and surrounding healthy regions. 
A)
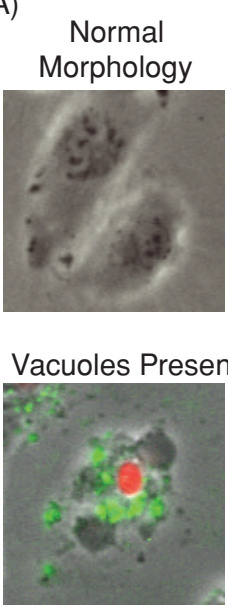

Condensed Nucleii

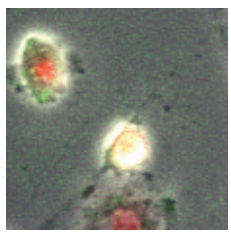

Rough

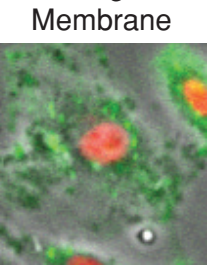

Blebbing

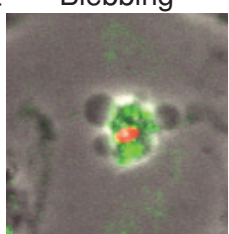

Irregular Nuclear Morphology

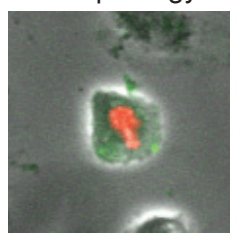

B) Low Intensity, Long Duration
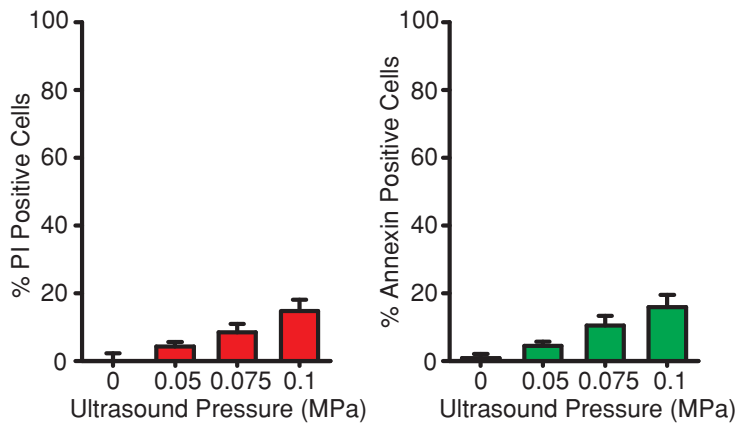

C) High Intensity, Short Duration

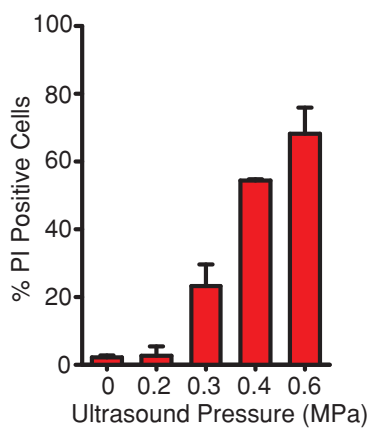

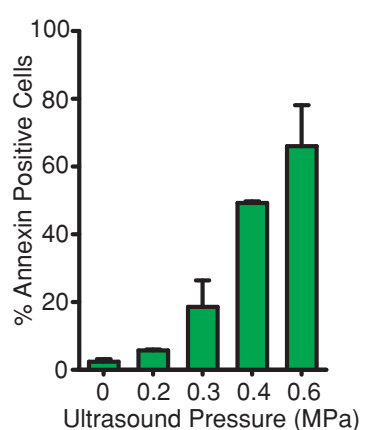

Figure 4. Effects of ultrasound excitation of microbubbles on HUVECs. A) Treated cells displayed a variety of apoptotic morphologies. Red signal corresponds to PI nuclear staining and green signal corresponds to annexinV staining. B) Ultrasound activation with low pressure (e.g., $<0.3 \mathrm{MPa}$ ) for a long treatment duration resulted in a low percentage of cells dying from apoptosis. C) Short ultrasound pulses at high pressure amplitudes (e.g., $0.4 \mathrm{MPa}$ and $0.6 \mathrm{MPa}$ ) resulted in significantly higher percentages of cells dying from apoptosis. Bars in $(B, C)$ denote mean $\pm S E M$ for $n>3$ independent experiments. Scale bars $\sim 20 \mu \mathrm{m}$.

Therefore, we next investigated the mechanism of sonoporation induced cell death. Using patterned microbubbles, we induced cell death in well-defined regions of the cell culture and stained with annexinV (to assay the appearance of phosphatidylserine on the cell surface) and PI (applied after ultrasound, to indicate loss of membrane integrity) (Figure 1I, Figure 4A). The annexinV-positive cells displayed a variety of morphologies indicative of apoptosis including changes in membrane appearance (roughening/ruffling), appearance of vacuoles and blebs and changes in nuclear morphology ${ }^{[32,33]}$ (Figure 4A). We tested the effects of two ultrasound regimes on cell apoptosis in our system. The first regime consisted of a low pressure ultrasound treatment over a long period (30 s) (Figure 4B). This treatment resulted in a low rate of acute apoptotic cell death, reaching a maximum of $\sim 20 \%$ at $0.1 \mathrm{MPa}$ as determined by $\mathrm{PI} /$ annexinV labeling. The second regime consisted of a high pressure, short $(8 \mu \mathrm{s})$ pulse of ultrasound (Figure $4 \mathrm{C})$. This treatment increased apoptotic cell death to $\sim 70 \%$ at $0.6 \mathrm{MPa}$.

In contrast to other forms of apoptosis, our treated cells were immediately positive for both the apoptosis marker annexinV and the secondary necrosis marker PI, with both markers generally localized to the same cells. This staining pattern may be due to the ability of microbubbles to simultaneously disrupt the plasma membrane and initiate apoptosis, as early apoptotic cells without membrane disruption would normally exclude PI. We hypothesized that apoptosis was initiated by an influx of extracellular calcium through ultrasound-induced membrane disruptions. We tested the effects of the levels of extracellular calcium on loss of cell viability/apoptosis in regions exposed to ultrasound treatment (Figure 5A-D). Higher acoustic pressure $(0.6 \mathrm{MPa})$ resulted in a higher percentage of cells labeled with PI and annexinV, as compared to lower acoustic pressure (0.3 MPa) (Figure 5A,B). This could be due to the fact that at higher acoustic pressures, more microbubbles were excited to initiate necrosis and apoptosis in more cells than at lower acoustic pressures. This is because smaller microbubbles usually require a higher acoustic pressure for cavitation to occur.

The extracellular calcium concentration had an important impact on both the percentage of PI positive cells and the percentage of annexinV positive cells. The percentage of dead cells (both PI- and annexinV-labeled) was markedly higher when the extracellular calcium concentration was increased to more than $5 \mathrm{mM}$, indicating the existence of a threshold for extracellular calcium above which there is an increase in the percentage of apoptotic cells. Interestingly, at lower calcium concentrations $(\leq 2 \mathrm{mM})$ and at $0.6 \mathrm{MPa}$ ultrasound pressure, the percentage of PI-positive cells $(\sim 60-65 \%)$ (Figure $5 \mathrm{~A}$ ) was greater than the percentage of annexinV-positive cells $(\sim 40$ $45 \%$ ) (Figure 5B). This indicates that there is a pool of cells that die directly from microbubble trauma (acute cell death) without initiating apoptosis. This trend was also observed at lower ultrasound pressures $(0.3 \mathrm{MPa})$, where the percentage of PI-positive cells $(\sim 20-38 \%)$ was greater than the percentage of annexinV-positive cells $(\sim 12 \%)$. However, at high enough 
A)

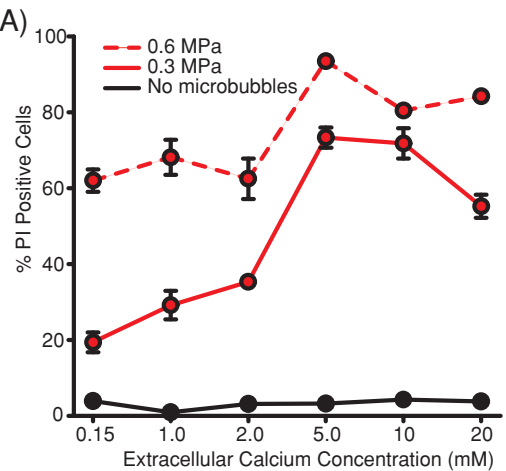

C) $0.15 \mathrm{mM} \mathrm{CaCl}_{2}+2 \mathrm{mM}$ EGTA
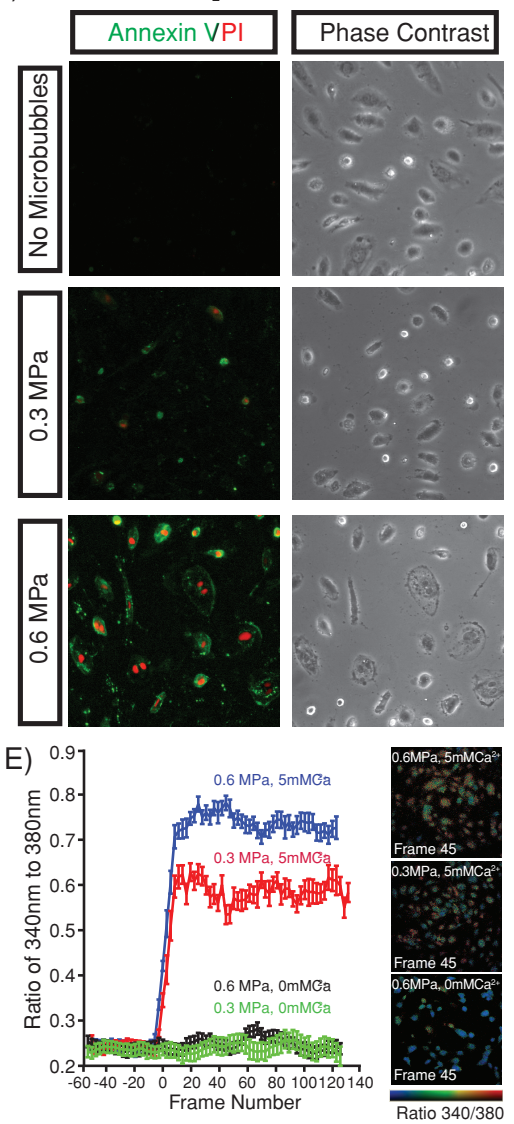

B)

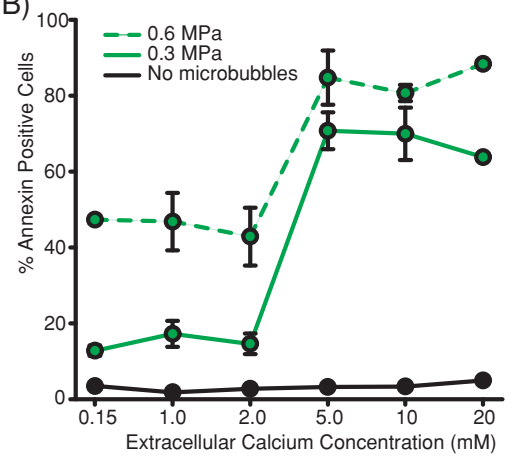

D)
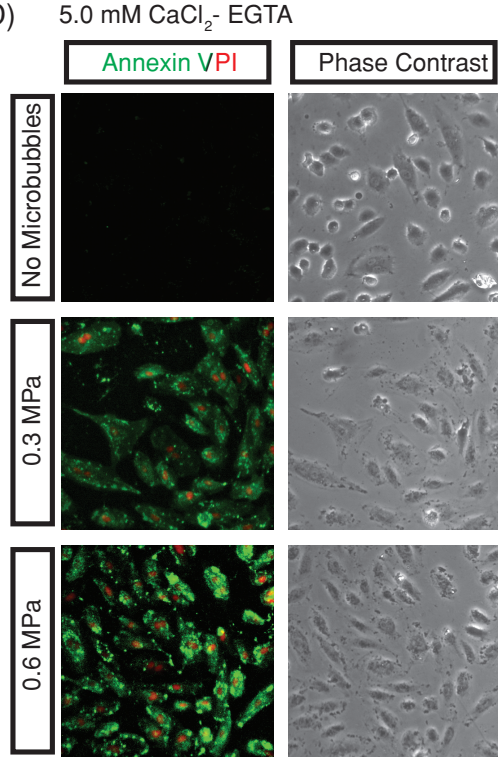

F)

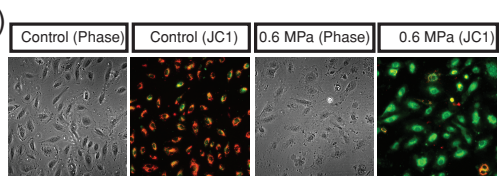

G)

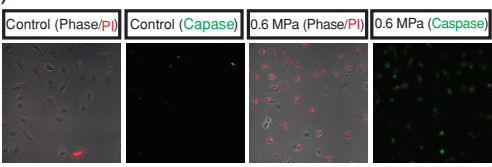

Figure 5. Effects of extracellular calcium concentration on apoptosis in microbubble patterned cultures. PI labeling of dead cells (A) and annexinV labeling of apoptotic cells (B) increased in the presence of high extracellular calcium ( $\geq 5 \mathrm{mM})$. Solid lines represent $0.3 \mathrm{MPa}$ ultrasound pressure, dashed lines represent $0.6 \mathrm{MPa}$ ultrasound pressure and black lines represent no ultrasound treatment. Bars denote mean $\pm \mathrm{SEM}$ for $\mathrm{n}>3$ independent experiments. C) Representative images of sonoporated cells in the presence of Cytomix buffer (no free extracellular calcium) for various ultrasound conditions. D) Representative image of sonoporated cells in the presence of $5 \mathrm{mM}$ calcium buffer for various ultrasound conditions. (E) Fura-2AM ratiometric calcium imaging of HUVECs treated with either 0 or $5 \mathrm{mM}$ calcium and either 0.3 or $0.6 \mathrm{MPa}$ ultrasound pressure, along with representative images taken at frame 45 of imaging (shortly after the ultrasound pulse). Bars denote mean \pm SEM. F) JCl staining for control (no ultrasound) and ultrasound-treated cells. Red staining indicates normal mitochondrial membrane potential and green staining indicates loss of membrane potential. G) Cell Event caspase staining for control (no ultrasound) and ultrasound-treated cells. Green nuclear staining indicates the activation of caspase in cells. All scale bars $\sim 40 \mu \mathrm{m}$.

calcium concentrations (e.g., $\geq 5 \mathrm{mM}$ ), almost all of the cells that were PI-positive were also annexinV-positive, suggesting either that increased extracellular calcium helped to reduce the number of cells with acute necrotic death, possibly by facilitating membrane repair for some cells, in accordance with what has been observed in other studies, ${ }^{[34-36]}$ or more likely, that elevated extracellular calcium concentration increased the number of cells undergoing apoptosis. This was true for both high and low pressure ultrasound conditions. Finally, at high calcium concentrations $(\geq 5.0 \mathrm{mM})$, low ultrasound pressures 
(0.3 $\mathrm{MPa}, 8 \mu \mathrm{s})$ were nearly as effective as high ultrasound pressures $(0.6 \mathrm{MPa}, 8 \mu \mathrm{s})$ at killing cells.

We performed ratiometric calcium imaging using Fura-2AM to confirm that calcium entered the cells as a result of microbubble excitation (Figure 5E). In the presence of extracellular calcium, there was a dramatic increase in the $340 / 380 \mathrm{~nm}$ ratio (corresponding to intracellular calcium concentration) that was observed immediately after the onset of ultrasound application, indicating that calcium was entering the cells driven by its concentration gradient. One consequence of rapid calcium influx is a loss of mitochondrial membrane potential, followed by further release of calcium from intracellular mitochondrial and endoplasmic reticulum stores, leading to propagation of intracellular pro-apoptotic signals. ${ }^{[37]}$ We confirmed that this occurred in the cells within the microbubble patterned regions by applying the JC1 ratiometric stain for mitochondrial membrane potential (Figure 5F). An increased level of green signal in the mitochondria indicated that mitochondrial function was disrupted by ultrasound induced calcium entry. Finally, to prove that cells entered the late stages of apoptosis, we assayed caspase activity using the Cell Event apoptosis detection kit (Figure 5G). Several hours after ultrasound treatment, almost every cell displayed a staining pattern indicative of caspase activity (cleavage of the Cell Event dye which subsequently labeled the nucleus). These results suggest a model whereby ultrasound excitation of microbubbles in the presence of high extracellular calcium leads to cell death that is almost exclusively apoptotic in nature. Our data also suggests that apoptosis is most likely mediated by influx of calcium into the cells which triggers the apoptosis cascade. Cells are unable to survive as this cascade initiates, resulting in simultaneous loss of membrane integrity with onset of apoptosis.

\section{Discussion}

We developed a new method for patterning microbubbles for controlled ultrasound-mediated cell membrane disruption (i.e., sonoporation). Our approach is easy to perform, can be applied to cells growing on a variety of substrates and offers the ability to apply multiplexed ultrasound/microbubble treatments to a small number of cells. We tested two types of commercially available microbubbles and found that streptavidin-coated (Targestar-SA) microbubbles performed better within our system. Although both Definity and Targestar-SA microbubbles possess a perfluorocarbon gas core bounded by a lipid shell, the difference in partitioning can be explained by the fact that the streptavidin on the surface of Targestar-SA microbubbles partitions to dextran. The ability to spatially pattern microbubbles enabled controlled and effective ultrasound treatment of cells. We demonstrated that patterning of microbubbles can be used to disrupt cell membranes of various cell types within defined regions, allowing intracellular entry of otherwise cell impermeant agents such as PI. The targeted areas could be monitored over several days to observe the cell responses in the damaged regions. We also demonstrated that the patterned microbubbles selectively induced apoptosis in subpopulations of cells following ultrasound treatment. This is relevant for researchers interested in apoptosis and useful as a testing platform for ultrasound/microbubble tissue disruption.

\subsection{Patterning of Microbubbles with ATPS}

Patterning techniques are frequently used in biomedical research for performing cell co-cultures, migration assays, wound healing assays, therapeutic targeting experiments and high throughput analyses. A variety of approaches have been developed for controlling placement of cells, including selective patterning of substrate materials, ${ }^{[38,39]}$ and direct cell printing with inkjets, stencils and more recently ATPSs. ${ }^{[7,9,40-43]}$ These technologies are useful for spatially organizing cells, but some cell types require patterning in situ (i.e., they are already growing on a surface). This is often the case for primary cells that have strict substrate requirements and a limited potential to divide in vitro. There are relatively few technologies that permit patterning on cells already growing as monolayers. ATPS micropatterning is capable of this type of patterning due to its biomolecule and particle partitioning ability. ${ }^{[6,8]}$ Here, we demonstrated that ATPSs can be used to deliver microbubbles to preformed monolayers of cells.

Physical disruption of the plasma membrane can be achieved by microinjection, hydrodynamic delivery, magnetotransfection, laser poration, ballistic delivery and electroporation, in addition to sonoporation. ${ }^{[44-47]}$ Among these methods, electroporation and sonoporation are two of the most effective physical methods for disrupting membrane integrity. ${ }^{[48-51]}$ However, neither technique is traditionally amenable to patterning. We demonstrated that spatial patterning of sonoporation could be achieved using ATPSs to confine microbubbles, which are known to be highly effective sonoporation facilitators.

Our ATPS microbubble patterning strategy provides several advantages for sonoporation. First, it provides an easy way to precisely place microbubbles in close vicinity to cells, thereby providing a controlled sonoporation platform. In our system, the microbubbles reside directly above the cells and are well dispersed in the XY dimensions, as confirmed by brightfield microscopy and our observations that microbubbles are in nearly the same focal plane as cells. This allowed us to overcome one of the main challenges in sonoporation research, the inherent unpredictability associated with free-floating microbubbles and controlling their impact on cells. Conventionally, sonoporation experiments have been conducted in centrifuge tubes or Petri dishes, where microbubbles are freely floating in the bulk solution. ${ }^{[52-55]}$ In these systems, the relative locations of the microbubbles and the cells are random and cannot be controlled. In some sonoporation experiments, cells are cultured inside thin chambers (e.g., Opticell). To ensure microbubbles are in contact with the cultured cells, these thin chambers are positioned upside down so that microbubbles float up to the adherent cells. ${ }^{[56-58]}$ However, this approach often leads to clustering of microbubbles at the boundaries of cells due to differences in the height of cells across the cell monolayer. In these systems, it is difficult, if not entirely impossible, to target a small region or sub-population of cells.

In our approach, the microbubbles can be easily positioned on any target cell population at controlled spatial locations. In 
most types of ultrasound experiments, long sonoporation treatment durations are used to maximize the chance of free-floating microbubbles impacting the cells. Since our approach minimizes free-floating microbubbles, we used short (microsecond) ultrasound durations; therefore, the microbubble dynamics are better controlled without large translational movements due to effects such as microstreaming.

Our system can be used for cells that grow on virtually any substrate, making this approach more versatile and adaptable. The ATPS medium itself imposes no acoustic discontinuity above the cells while confining the bubbles within a fluid (this is problematic for most microdevices). In addition, our approach reduces the amount of reagent and the number of microbubbles by as much as 10 000-fold because microbubbles are confined in $0.1 \mu \mathrm{L}$ DEX droplets instead of $10 \mathrm{~mL}$ solutions used in conventional sonoporation experiments in a dish or similar container. Since we use small amounts of microbubbles and can generate well defined patterns, this approach is highly amenable to multiplexing ultrasound parameters, the microbubble type/ concentration, and the concentration and type of biomolecule present in the extracellular space. The final advantage of our system relates to the need for antibody/microbubble conjugation. For targeted microbubble applications, antibody conjugation is needed to ensure microbubble targeting and attachment to the cell surface. Our approach does not require antibodies for targeting cells because the DEX droplets confine the microbubbles at the cell surface. This allows the same microbubbles to be used with any cell type without any modification.

\subsection{Implications for Apoptosis Research}

Ultrasound-excited microbubbles that are capable of generating significant physical impact on cells at the micro- and nano-scales provide a unique tool for manipulation of cells. Patterning of microbubbles using ATPSs provides a novel system with great potential for new applications. In this study, we demonstrated a novel application of ATPS microbubble patterning for selectively inducing apoptosis. A number of studies have shown sonoporation induced apoptosis in cells, ${ }^{[52,59-61]}$ although the mechanisms are not completely understood. Moreover, in sonoporation-mediated gene delivery experiments, a large number of cells are often damaged or killed by ultrasound-activated microbubbles, although the number of dead cells and the mechanism of their death (i.e., apoptosis vs necrosis) are not always clearly reported in the literature. In our study, the spatial localization and patterning of microbubbles using ATPS enabled detailed investigation of ultrasound excitation of microbubbles and their effects on the cells. With ATPS microbubble patterning, we induced a high proportion of cells to enter apoptosis in targeted areas within a monolayer by inducing calcium influx into the cells during sonoporation. Based on our previous experiments using DEX as a delivery vehicle for lipofection agents, the DEX and PEG polymers have little to no effect on cell viability. Therefore, apoptosis was mediated by microbubble-induced membrane damage and calcium influx, as evidenced by a dose response to extracellular calcium. This example illustrates that our technology of ATPS microbubble patterning may be used as a potential platform for studying apoptosis through transmembrane delivery of ionic or chemical initiators of apoptosis. Since our technique facilitates multiplexing of both microbubble condition and ultrasound treatment for testing the effects of sonoporation on cell viability, this may be useful for studying localized cell injury or apoptosis, while testing a wide range of treatments or acquiring a large number of independent observations. ${ }^{[62,63]}$

Apoptotic cells are recognized and removed by resident macrophages in tissue; whereas damage caused by necrotic injury is not recognized by the immune system and can lead to toxicity in the surrounding tissue. ${ }^{[64]}$ Since apoptosis is highly localized to specific cell populations in our system, ATPS microbubble patterning for ultrasound excitation may serve as a useful test system for investigating the interactions of apoptotic cells with surrounding cells, including the ability of healthy cells to migrate into apoptotic regions and remove debris. The effect that dying cells have on neighboring healthy populations can also be readily investigated in our system. Furthermore, by using ATPS and microbubbles to locally kill cells it will be possible to perform well controlled wound healing assays without physical removal of the damaged region. In our system, the wounded region consisting of both apoptotic and necrotic cells may represent a more physiologically relevant in vitro model for wound healing.

\subsection{Future Directions}

It will be useful to design easier to use setups to dispense the DEX droplets and apply ultrasound excitation to microbubbles. Development of new microbubbles that partition better in DEX can also help to improve and expand this technology. Finally, our technology may serve as a useful high throughput testing platform to investigate the mechanisms for various microbubble-potentiated ultrasound applications such as ultrasound mediated drug delivery for cancer treatment, ${ }^{[65]}$ as well as ultrasound tissue ablation, where fractionation, necrosis and enhanced apoptosis have been identified as factors beneficial for treatment. ${ }^{[66-68]}$

\section{Experimental Section}

ATPS Formulation: ATPSs were formulated from DEX (ranging in molecular weight from $3500 \mathrm{kDa}$ to $500000 \mathrm{kDa}$; Pharmacosmos, Denmark) and PEG 35000 kDa (Sigma, St. Louis, MO). These polymers were reconstituted as aqueous solutions in various buffered media. The initial formulations tested were near critical point concentrations of $9 \%$ DEX $3500 \mathrm{kDa} / 10 \%$ PEG $35000 \mathrm{kDa}, 7 \%$ DEX $10000 \mathrm{kDa} / 7 \%$ PEG $35000 \mathrm{kDa}$, and 3.2\% DEX $500000 \mathrm{kDa} / 2.5 \%$ PEG $35000 \mathrm{kDa}$ in their fully equilibrated forms. For experiments that required additional reagents in the ATPS solutions, concentrations slightly higher than the critical points were used (e.g., stock solutions of 16\% DEX 10000 $\mathrm{kDa} / 16 \%$ PEG $35000 \mathrm{kDa}$, corresponding to a solution of approximately $8 \%$ DEX $10000 \mathrm{kDa} / 8 \%$ PEG $35000 \mathrm{kDa}$ after full equilibration. ATPSs formed from stock solutions of $8 \%$ DEX $10000 \mathrm{kDa} / 16 \%$ PEG 35000 $\mathrm{kDa}$ and 4\% DEX $10000 \mathrm{kDa} / 16 \%$ PEG $35000 \mathrm{kDa}$ were also tested.

Microbubble Partitioning: Two types of commercially available ultrasound contrast agent microbubbles were used in this study: Definity (Lantheus Medical Imaging, Billerca, MA) and Targestar-SA (Targeson, San Diego, CA). Definity microbubbles are composed of 
a $\mathrm{C}_{3} \mathrm{~F}_{8}$ gas core, encapsulated by a phospholipid shell. Targestar-SA is a perfluorocarbon microsphere, encapsulated with a lipid shell coated with streptavidin, which permits biotinylated ligands to bind to its surface via biotin-streptavidin conjugation chemistry. The affinities of different microbubbles for the above phase systems were tested by mixing stock solutions of microbubbles in equal concentrations of twice-concentrated solutions of PEG and DEX in a microcentrifuge tube. The phase systems were then allowed to separate by gravity at room temperature. Once the phase systems reached equilibrium ( $\sim 10 \mathrm{~min})$, samples were taken from the top (PEG) and bottom (DEX) phases along with the PEG/DEX interface and diluted below the critical point $1: 10$ in PBS) to remove any residual PEG/DEX droplets that could be mistaken for microbubbles. Microbubbles were placed on a hemocytometer and imaged under brightfield illumination using a 10x objective. Images were imported into image) and numbers of microbubbles were automatically quantified using the automated nuclei counting plugin (Center for BioImage Informatics, UCSB). Partitioning was measured for Definity lipid coated microbubbles, Targestar-SA microbubbles (without antibody) and Targestar-SA microbubbles conjugated to biotinylated IgG by counting the numbers of microbubbles in each phase. Partitioning was also investigated by placing the microbubbles within non-equilibrated twiceconcentrated solutions of DEX which were then deposited into PEG as $1 \mu \mathrm{L}$ droplets. The movement of microbubbles within the DEX droplets was monitored using video microscopy.

Cell Culture: Human umbilical vein endothelial cell (HUVEC) preparations (passage number 3-7) were plated one day prior to the experiment onto tissue culture treated $35 \mathrm{~mm}^{2}$ glass bottom dishes. NIH3T3, MDA231 and HeLa cells were cultured under standard conditions and then either seeded separately or as mixtures (co-cultures) in HUVEC medium in $35 \mathrm{~mm}^{2}$ glass bottom dishes one day prior to the experiment. A seeding density of 200 cells $/ \mathrm{mm}^{2}$ was used to ensure $\sim 80 \%$ confluence the following day. Cultures were maintained in a humidified incubator at $37^{\circ} \mathrm{C}$, with $5 \% \mathrm{CO}_{2}$. For experimental treatments, cultures were removed from the incubator, covered with a solution of $16 \%$ PEG, patterned with DEX/microbubbles and immediately treated by ultrasound. After $10 \mathrm{~min}$ incubation, the ATPS was removed through 3-5 consecutive washes in PBS. The cells were then either reintroduced to culture medium and returned to standard incubation conditions for later analysis or immediately stained and imaged.

Microbubble Preparation and ATPS Micropatterning: Microbubbles $\left(\sim 1 \times 10^{9}\right.$ particles per $\left.\mathrm{mL}\right)$ were incorporated into the DEX phase by mixing a solution of $32 \%$ DEX $10000 \mathrm{kDa}$ with an equal volume of microbubbles to obtain a solution of $\sim 0.5 \times 10^{9}$ microbubbles $/ \mathrm{mL}$ in $16 \%$ DEX, representing the optimal microbubble concentration. Solutions with 10 -fold and 100 -fold lower microbubble concentrations were also tested. The microbubble solutions were loaded into capillary needles that were positioned above the PEG coated cells. Capillary tips were lowered into the PEG and droplets of DEX containing microbubbles $(\sim 0.05-0.1 \mu \mathrm{L}$ in volume) were dispensed in the desired patterns above the cells. These droplets sank vertically in the PEG solution, where they came into contact with the cells and spread on the cell monolayer. Once the final droplet was in place, the micropatterned cultures were ready for ultrasound exposure.

Experimental Setup for Ultrasound Application: The experimental setup consisted of a microscope equipped with a non-focused circular ultrasound transducer (center frequency $1.25 \mathrm{MHz}$, active element diameter $0.6 \mathrm{~cm}$ ) (Advanced Devices, Wakefield, MA, USA) that was driven by a function generator (33250A, Agilent Technologies, Palo Alto, CA, USA) and an amplifier (75A250, Amplifier Research, Souderton, PA, USA). The transducer was calibrated in free field in water using a calibrated $40 \mu \mathrm{m}$ needle hydrophone (HPM04/1, Precision Acoustics, Dorchester, UK). The transducer was positioned at 45 degrees (to avoid standing wave buildup and accommodate simultaneous microscopic imaging) with its active surface $\sim 7.5 \mathrm{~mm}$ (Rayleigh distance) from the cell culture surface such that the maximum ultrasonic pressure was focused on the cells. This configuration allowed us to simultaneously apply ultrasound and microscopically observe microbubbles. Ultrasound excitation of microbubbles was monitored by a high speed camera
(Photron FASTCAM SA1, San Diego, CA) at a frame rate of 20000 frames $\mathrm{s}^{-1}$. A cooled CCD camera (Photometrics QuantEM, Tucson, AZ, USA), a monochromator with $5 \mathrm{~nm}$ bandpass (DeltaRAM X, PTI, Birmingham, N), USA) and a polychroic filter (73000v2, Chroma, Rockingham, VT, USA) were used to perform ratiometric epifluorescence and standard epifluorescence imaging of molecules delivered by sonoporation. The ultrasound treatments were applied either as single $8 \mu \mathrm{sec}$ pulses or as continuous ( $30 \mathrm{~s}$ ) wave forms that varied in peak acoustic pressure from $0.05 \mathrm{MPa}$ to $0.6 \mathrm{MPa}$.

Apoptosis and Calcium Influx: To investigate whether calcium influx during sonoporation plays a role in sonoporation triggered apoptosis in our system, we used PEG solutions containing various calcium concentrations either in a buffer designed to mimic the intracellular ion concentration of cells (Cytomix) ${ }^{[69]}$ or in PBS/DPBS. Calcium concentrations ranged from 0 to $20 \mathrm{mM}$.

Cells were exposed to either 0.3 or $0.6 \mathrm{MPa}$ peak acoustic pressures and allowed to recover for $15 \mathrm{~min}$ at room temperature before washing in PBS. Cells were then washed in annexinV binding buffer and incubated with a solution containing Alexa 488-annexinV (Invitrogen) and $\mathrm{PI}$ to detect apoptosis. Regions with microbubbles were compared to regions without microbubbles and the total number of $\mathrm{PI}$-positive and annexinV-positive cells was quantified as before for each condition, with the exception that PI was added after ultrasound treatment and used as a dead cell marker. Ratiometric imaging using Fura-2 AM (Invitrogen) was used to confirm that changes in intracellular calcium had occurred following ultrasound treatment. For ratiometric calcium imaging, cells were loaded with Fura2-AM before ultrasound by incubating the cells with Fura2-AM and Pluronic F-127 (0.05\% v/v of $10 \% \mathrm{w} / \mathrm{v})$ for $60 \mathrm{~min}$ at $37^{\circ} \mathrm{C}$. The cells were then washed 3 times to remove extracellular Fura-2AM. ${ }^{[70]}$ To quantify intracellular calcium concentration following ultrasound, the ratio of signal at $340 / 380 \mathrm{~nm}$ was calculated for each treated cell.

Viability Assays: To analyze cell viability as a function of ultrasound/ calcium-induced apoptosis, we used several independent tests. PI staining, applied after ultrasound treatment, revealed cells that were not able to reseal their membranes after sonoporation, while annexinV labeling was used as an early stage marker for apoptosis. Phase contrast images were used to quantify the total number of cells in each image and the percentage of annexinV- and PI-positive cells was calculated for various ultrasound and calcium conditions. Phase contrast and $\mathrm{PI}$ images also allowed us to qualitatively inspect the status of cells in terms of general morphology, membrane features and nuclear morphology. JC1 ratiometric staining (Invitrogen) was used to confirm that mitochondrial membrane potential was disrupted in the apoptotic cells. Cell Event caspase stain (Invitrogen) was used to confirm that cells entered the late stages of apoptosis.

Data Analysis: Image J and MetaFluor Analyst (Molecular Devices, Downingtown, PA, USA) photometric software were used for image analysis. Mosaic and live cell images were captured, compiled and stitched using Zeiss Axiovision software. Statistical analyses were carried out using Sigmaplot with Sigmastat. All graphs are presented as mean values \pm standard error of the mean.

\section{Supporting Information}

Supporting Information is available from the Wiley Online Library or from the author.

\section{Acknowledgements}

J.P.F., Z.F., and A.S. contributed equally to this work. S.T. and C.X.D. acknowledge funding from the Beyster foundation. S.T. acknowledges funding from NIH (CA136829 and HL084370), Coulter Foundation, and NSF (IIP 1243080), and C.X.D. acknowledges funding from NIH (CA116592). We thank Yubing Sun and Shinuo Weng from Dr. Jianping 
Fu's lab at University of Michigan for their assistance with mosaic and live cell imaging.

Received: November 12, 2012 Published online: February 6, 2013

[1] A. L. Hook, H. Thissen, N. H. Voelcker, Trends Biotechnol. 2006, 24, 471.

[2] S. Takayama, E. Ostuni, P. LeDuc, K. Naruse, D. E. Ingber, G. M. Whitesides, Nature 2001, 411, 1016.

[3] S. Takayama, E. Ostuni, P. LeDuc, K. Naruse, D. E. Ingber, G. M. Whitesides, Chem. Biol. 2003, 10, 123.

[4] T. Jain, J. Muthuswamy, Biosens. Bioelectron. 2007, 22, 863.

[5] J. K. Valley, S. Neale, H. Y. Hsu, A. T. Ohta, A. Jamshidi, M. C. Wu, Lab Chip 2009, 9, 1714.

[6] H. Tavana, A. Jovic, B. Mosadegh, Q. Y. Lee, X. Liu, K. E. Luker, G. D. Luker, S. J. Weiss, S. Takayama, Nat. Mater. 2009, 8, 736.

[7] H. Tavana, B. Mosadegh, S. Takayama, Adv. Mater. 2010, 22, 2628.

[8] P. A. K. Albertsson, Partition of cell particles and macromolecules: separation and purification of biomolecules, cell organelles, membranes, and cells in aqueous polymer two-phase systems and their use in biochemical analysis and biotechnology, Wiley, New York 1986.

[9] Y. Fang, J. P. Frampton, S. Raghavan, R. Sabahi-Kaviani, G. Luker, C. X. Deng, S. Takayama, Tissue Eng., C 2012, 18, 647.

[10] T. Yaguchi, M. Dwidar, C. K. Byun, B. Leung, S. Lee, Y. K. Cho, R. J. Mitchell, S. Takayama, Biomacromolecules 2012, 13, 2655.

[11] P. Marmottant, S. Hilgenfeldt, Nature 2003, 423, 153.

[12] M. Postema, A. van Wamel, C. T. Lancee, N. de Jong, Ultrasound Med. Biol. 2004, 30, 827.

[13] P. Prentice, A. Cuschieri, K. Dholakia, M. Prausnitz, P. Campbell, Nat. Phys. 2005, 1, 107

[14] C. D. Ohl, M. Arora, R. Ikink, N. de Jong, M. Versluis, M. Delius, D. Lohse, Biophysical J. 2006, 91, 4285.

[15] A. van Wamel, K. Kooiman, M. Harteveld, M. Emmer, F. J. ten Cate, M. Versluis, N. de Jong, J. Controlled Release 2006, 112, 149.

[16] D. L. Miller, S. V. Pislaru, J. E. Greenleaf, Somatic Cell Mol. Genet. 2002, 27, 115.

[17] N. Kudo, K. Okada, K. Yamamoto, Biophys. J. 2009, 96, 4866.

[18] K. W. Ferrara, M. A. Borden, H. Zhang, Acc. Chem. Res. 2009, 42, 881.

[19] A. L. Klibanov, Med. Biol. Eng. Comput. 2009, 47, 875

[20] J. R. Lindner, Nat. Rev. Drug Discovery 2004, 3, 527.

[21] X. Wang, C. E. Hagemeyer, J. D. Hohmann, E. Leitner, P. C. Armstrong, F. Jia, M. Olschewski, A. Needles, K. Peter, I. Ahrens, Circulation 2012, 125, 3117.

[22] K. Kooiman, M. Foppen-Harteveld, A. F. van der Steen, N. de Jong, J. Controlled Release 2011, 154, 35.

[23] G. Korpanty, S. Chen, R. V. Shohet, J. Ding, B. Yang, P. A. Frenkel, P. A. Grayburn, Gene Ther. 2005, 12, 1305.

[24] J. M. Warram, A. G. Sorace, R. Saini, A. V. Borovjagin, K. Hoyt, K. R. Zinn, Cancer Gene Ther. 2012, 19, 545.

[25] Z. Fan, H. Liu, M. Mayer, C. X. Deng, Proc. Natl. Acad. Sci. USA 2012, 109, 16486.

[26] P. A. Dayton, K. E. Morgan, A. L. Klibanov, G. Brandenburger, K. R. Nightingale, K. W. Ferrara, IEEE Trans. Ultrason. Ferroelectr. Freq. Control 1997, 44, 1264.

[27] V. Bjerknes, Fields of force; supplementary lectures, applications to meteorology; a course of lectures in mathematical physics delivered December 1 to 23, 1905, The Columbia University Press, New York 1906.

[28] J. C. Yarrow, Z. E. Perlman, N. J. Westwood, T. J. Mitchison, BMC Biotechnol. 2004, 4, 21.
[29] C. C. Liang, A. Y. Park, J. L. Guan, Nat. Protoc. 2007, 2, 329.

[30] C. R. Keese, J. Wegener, S. R. Walker, I. Giaever, Proc. Natl. Acad. Sci. USA 2004, 101, 1554.

[31] F. Q. Nie, M. Yamada, J. Kobayashi, M. Yamato, A. Kikuchi, T. Okano, Biomaterials 2007, 28, 4017.

[32] S. L. Fink, B. T. Cookson, Infect. Immun. 2005, 73, 1907.

[33] U. Ziegler, P. Groscurth, News Physiol. Sci. 2004, 19, 124.

[34] Y. Zhou, J. Shi, J. Cui, C. X. Deng, J. Controlled Release 2008, 126, 34

[35] C. X. Deng, F. Sieling, H. Pan, J. Cui, Ultrasound Med. Biol. 2004, 30, 519.

[36] P. L. McNeil, M. Terasaki, Nat. Cell Biol. 2001, 3, E124.

[37] S. Orrenius, B. Zhivotovsky, P. Nicotera, Nat. Rev. Mol. Cell Biol. 2003, 4, 552

[38] D. Falconnet, G. Csucs, H. M. Grandin, M. Textor, Biomaterials 2006, 27, 3044.

[39] J. Y. Lim, H. J. Donahue, Tissue Eng. 2007, 13, 1879.

[40] B. R. Ringeisen, C. M. Othon, J. A. Barron, D. Young, B. J. Spargo, Biotechnol. J. 2006, 1, 930

[41] D. Wright, B. Rajalingam, S. Selvarasah, M. R. Dokmeci, A. Khademhosseini, Lab Chip 2007, 7, 1272.

[42] H. Tavana, B. Mosadegh, P. Zamankhan, J. B. Grotberg, S. Takayama, Biotechnol. Bioeng. 2011, 108, 2509.

[43] H. Tavana, S. Takayama, Biomicrofluidics 2011, 5, 13404.

[44] M. S. Al-Dosari, X. Gao, AAPS J. 2009, 11, 671

[45] S. Mehier-Humbert, R. H. Guy, Adv. Drug Delivery Rev. 2005, 57, 733.

[46] J. Villemejane, L. M. Mir, Brit. J. Pharmacol. 2009, 157, 207.

[47] D. J. Wells, Gene Ther. 2004, 11, 1363.

[48] J. L. Lee, C. W. Lo, S. M. Ka, A. Chen, W. S. Chen, J. Controlled Release 2012, 160, 64

[49] R. Suzuki, Y. Oda, N. Utoguchi, K. Maruyama, J. Controlled Release 2011, 149, 36

[50] D. J. Wells, Cell Biology Toxicology 2010, 26, 21.

[51] L. H. Treat, N. McDannold, N. Vykhodtseva, Y. Zhang, K. Tam, K. Hynynen, Int. J. Cancer 2007, 121, 901.

[52] W. Zhong, W. H. Sit, J. M. Wan, A. C. Yu, Ultrasound Med. Biol. 2011, 37, 2149.

[53] A. Lawrie, A. F. Brisken, S. E. Francis, D. C. Cumberland, D. C. Crossman, C. M. Newman, Gene Ther. 2000, 7, 2023.

[54] S. Zhang, J. Cheng, Y. X. Qin, PLoS One 2012, 7, 38343.

[55] R. Karshafian, P. D. Bevan, R. Williams, S. Samac, P. N. Burns, Ultrasound Med. Biol. 2009, 35, 847.

[56] L. C. Phillips, A. L. Klibanov, B. R. Wamhoff, J. A. Hossack, Ultrasound Med. Biol. 2010, 36, 1470.

[57] B. D. Meijering, L. J. Juffermans, A. van Wamel, R. H. Henning, I. S. Zuhorn, M. Emmer, A. M. Versteilen, W. J. Paulus, W. H. van Gilst, K. Kooiman, N. de Jong, R. J. Musters, L. E. Deelman, O. Kamp, Circ. Res. 2009, 104, 679.

[58] J. L. Tlaxca, C. R. Anderson, A. L. Klibanov, B. Lowrey, J. A. Hossack, J. S. Alexander, M. B. Lawrence, J. J. Rychak, Ultrasound Med. Biol. 2010, 36, 1907.

[59] H. Honda, T. Kondo, Q. L. Zhao, L. B. Feril Jr., H. Kitagawa, Ultrasound Med. Biol. 2004, 30, 683.

[60] M. A. Hassan, P. Campbell, T. Kondo, Drug Discovery Today 2010, $15,892$.

[61] L. B. Feril Jr., T. Kondo, Q. L. Zhao, R. Ogawa, K. Tachibana, N. Kudo, S. Fujimoto, S. Nakamura, Ultrasound Med. Biol. 2003, 29, 331.

[62] D. Huh, H. Fujioka, Y. C. Tung, N. Futai, R. Paine, 3rd, J. B. Grotberg, S. Takayama, Proc. Natl. Acad. Sci. USA 2007, 104, 18886.

[63] N. J. Douville, P. Zamankhan, Y. C. Tung, R. Li, B. L. Vaughan, C. F. Tai, J. White, P. J. Christensen, J. B. Grotberg, S. Takayama, Lab Chip 2011, 11, 609. 
[64] G. Wickman, L. Julian, M. F. Olson, Cell Death Differ. 2012, 19, 735.

[65] G. J. Czarnota, R. Karshafian, P. N. Burns, S. Wong, A. Al Mahrouki, J. W. Lee, A. Caissie, W. Tran, C. Kim, M. Furukawa, E. Wong, A. Giles, Proc. Natl. Acad. Sci. USA 2012, 109, 2033.

[66] Z. Xu, Z. Fan, T. L. Hall, F. Winterroth, J. B. Fowlkes, C. A. Cain, Ultrasound Med. Biol. 2009, 35, 245.
[67] Z. Xu, T. L. Hall, J. B. Fowlkes, C. A. Cain, J. Acoust. Soc. Am. 2007, 122, 229.

[68] J. E. Kennedy, Nat. Rev. Cancer 2005, 5, 321.

[69] M. J. van den Hoff, A. F. Moorman, W. H. Lamers, Nucleic Acids Res. 1992, 20, 2902.

[70] Z. Fan, R. E. Kumon, J. Park, C. X. Deng, J. Controlled Release 2010, $142,31$. 